\title{
STABILITY AND CONVERGENCE ANALYSIS OF SMOKING IMPACT IN SOCIETY WITH ALGORITHM ASPECTS
}

\author{
AQEEL AHMAD*, MARYAM SHAHID, MUHAMMAD FARMAN, M.O. AHMAD \\ Department of Mathematics and Statistics, The University of Lahore, Lahore, Pakistan \\ *Corresponding author: aqeelahmad.740@gmail.com
}

\begin{abstract}
In this manuscript, an epidemic model employed the dynamics of drugs usage among adults. Among smokers, often the desire to quit smoking arises. A large number of smokers attempt to quit, but only a few of them are successful. A non-linear mathematical model is employed to study and assess the dynamics of smoking and its impact on public health in a community. We prove the essential properties, bounded, positivity and well-posed, also local and global stability analysis has been made for the epidemic model. The sensitivity analysis of the model is provided by threshold or reproductive number as well as analyzed qualitatively. We develop an unconditionally convergent nonstandard finite difference scheme by applying Mickens approach $\phi(h)=h+O\left(h^{2}\right)$ instead of $h$ to control the spread of bad impact in society. Finally numerical simulations are also established to investigate the influence of the system parameters on the spread of the smoking impact in society.
\end{abstract}

\section{INTRODUCTION}

The scope of mathematics includes mathematical modeling and esoteric mathematics. The flow of work, process, predictions and outcomes can easily be measured with the help of mathematical concepts and theories. Therefore, biologists are now extremely dependent on mathematics. Mathematical modeling of biological sciences has been conducted [1-3]. The relationship between simple mathematical modeling involves biological system, integer order differential equations that show their dynamics and complex system which describes their changing of structure. The nonlinearity and multi-scale behaviors in mathematical modeling

Received 2019-04-11; accepted 2019-05-14; published 2019-07-01.

2010 Mathematics Subject Classification. 37M05,92B05.

Key words and phrases. stability analysis; qualitative analysis; convergence analysis; sensitivity analysis.

(C)2019 Authors retain the copyrights of their papers, and all open access articles are distributed under the terms of the Creative Commons Attribution License. 
describe the mutual relationship between parameters [4]. In last few decades, many biological models were studied in detail by using classical derivative [5-8].

Smoking is the large problem in the entire world. Despite overwhelming facts about smoking, it is still a very bad habit which is widely spread and accepted socially. Smoking is a bad habit in which a substance is burned and the resulting smoke breathed to taste and absorbed into the bloodstream [14]. Tobacco pandemic is one of the largest public medicinal threats to the world has ever faced, as it puts to death up to half of its users. Smoking kills about six million people each year of whom more than five million are ex-smokers and smokers and over 500,000 are nonsmokers revealed to second-hand smoke. Tobacco users who pass away prematurely deprive their families of earnings, lift the cost of fitness care and through them in deep financial crisis. The World Health Organization forecasts that by 2030, ten million persons will pass away every year due to tobacco associated illnesses. Numerous mathematical models for smoking have been developed in the last few years $[9,13]$.

Epidemiology is related with the spread of diseases in population and the major factors that effects the transmission of diseases [15]. The subject of smoking is one of the interesting areas in study of epidemiology. There is a lot of work that has been done on the smoking epidemics models [16-18]. Mathematical models are established to understand the dynamics of the contagious diseases. These models are divided into compartments with population are called compartmental models. The assumptions of transfer data from one to another compartment depends upon the nature and time rate. The major idea in these models is that the people will start to live healthy in a community. Diseases may effect the healthy peoples but effected one could become healthy again in the community [19-22]. Different numerical technique can be employed for epidemic model to analyse the data for control strategy. Different technique converges conditionally and diverge for large step size for epidemic model but NSFD scheme converges unconditionally.

In this paper, we investigate the stability and qualitative analysis of the smoking model. An unconditionally convergent nonstandard finite difference scheme has been presented to obtain solution of smoking model. The analysis of two different states disease free and endemic equilibrium which means the disease dies out or persist in a population has been made by finding reproductive number. Numerical results are presented graphically to show the dynamics of the model.

\section{Materials and Method}

The concept of mathematical modeling has been prolonged to define the stability and qualitative features of giving up smoking models from 2000 [26-28]. Many researchers considered various smoking models like [23], in which they examined numerous classes of smokers (potential smokers $P(t)$, smokers $S(t)$, and quit smokers $Q(t)$. Then [24] modified the proposed model and introduced a new class named as chain smokers. Later on, Zeb et al. [25] studied a smoking model in which they argued local and global stability of the model and 
its general solutions in which the collaboration between occasional and potential smokers occurs. In [26] the author presented a modified smoking model in which the dynamic behavior has been discussed numerically. The proposed smoking model [8] in the form of system of differential equation is given as:

$$
\begin{gathered}
\frac{d P}{d t}=b N-\beta_{1} L P-\left(d_{1}+\mu\right) P+\tau Q \\
\frac{d L}{d t}=\beta_{1} L P-\beta_{2} L S-\left(d_{2}+\mu\right) L \\
\frac{d S}{d t}=\beta_{2} L S-\left(\gamma+d_{3}+\mu\right) S \\
\frac{d Q}{d t}=\gamma S-\left(\tau+d_{4}+\mu\right) Q, \\
\frac{d N}{d t}=(b-\mu) N-\left(d_{1} P+d_{2} L+d_{3} S+d_{4} Q\right)
\end{gathered}
$$

with initial conditions $P(0)=n_{1}, L(0)=n_{2}, S(0)=n_{3}, Q(0)=n_{4}, N(0)=n_{5}$, where $P, L, S, Q$ and $N$ represents the smoking status of time dependent sub-compartments $b, \mu, \gamma$ and $\tau$ are the parameter involved $\beta_{1}$ and $\beta_{2}$ are the transmission coefficient $d_{1}, d_{2}, d_{3}$ and $d_{4}$ are the death rates of the sub-compartments of the smoking model.

\section{STABility OF THE MODEL}

In this section, we find the basic reproduction number and stability of the model. We prove that our model is locally and globally stable for disease-free-equilibrium. Since all our model parameters are positive or non-negative, it is important to show that all state variables remain positive or non-negative for all positive initial conditions for 0 . From our model equation, we have

$$
\left.\frac{d N}{d t}=(b-\mu) N-\left(d_{1} P+d_{2} L+d_{3} S+d_{4} Q\right) \leq b-\mu\right) N
$$

The closed set is

$$
D=\left(P, L, Q, S, N \in R_{+}^{5}: N \leq \frac{b}{\mu}\right)
$$

Theorem 1: The closed set D is bounded and positive invariant.

Proof: $\frac{d N}{d t} \leq b N-\mu N$,

So $N$ is bounded above by $b N-\mu N$, Hence $\frac{d N}{d t}<0$ and $t>\frac{b}{\mu}$.

On simplification we have, $N(t) \leq N(0) e^{-\mu t}+\frac{b}{\mu}\left(1-e^{-\mu t}\right)$.

As $t \rightarrow \infty, e^{-\mu} \rightarrow 0$, So $\lim _{t \rightarrow \infty} N(t) \leq \frac{b}{\mu}$

Thus, $D$ is bounded and positively invariant in $R_{+}^{5}$

Theorem 2: Assume that model $(2.1-2.4)$ has global solution corresponding to non-negative initial 
condition then solution is non negative all times. .

Proof:Assume $P(0) \geq 0, L(0) \geq 0, Q(0) \geq 0$, equation can be written as

$$
\begin{gathered}
\frac{d P}{d t}=b N-\left(\beta_{1} L+d_{1}+\mu\right) P \\
\frac{d P}{d t}=b N-D P
\end{gathered}
$$

This linear first order differential equation in $P$ which has solution

$$
P=P(0) \exp \left(\int_{0}^{t}-A(P) d p+\exp \int_{0}^{t}-A(P) d t \times 0 \int_{0}^{t} \pi \exp \left(\int_{0}^{u}-A(w) d w\right)\right) d u \geq 0
$$

Hence $P \geq 0$ for all $t \geq 0$, regarding the non-negativity of the remaining variables, we consider the subsystem

$$
\begin{gathered}
\frac{d L}{d t}=\beta_{1} L P-\beta_{2} L S-\left(d_{2}+\mu\right) L \\
\frac{d S}{d t}=\beta_{2} L S-\left(\gamma+d_{3}+\mu\right) S \\
\frac{d Q}{d t}=\gamma S-\left(\tau+d_{4}+\mu\right) Q,
\end{gathered}
$$

This can be written in matrix form

$$
\begin{aligned}
& \frac{d Y}{d t}=M Y(t)+B(t) \\
& \text { where } Y(t)=\left[\begin{array}{c}
L(t) \\
S(t) \\
Q(t)
\end{array}\right], M=\left[\begin{array}{ccc}
-\beta_{1}-\left(d_{2}+\mu\right) & 0 & 0 \\
0 & -\left(\gamma+d_{3}+\mu\right) & 0 \\
0 & \gamma & -\left(\tau+d_{4}+\mu\right)
\end{array}\right] \\
& \text { and } B(t)=\left[\begin{array}{c}
0 \\
0 \\
\gamma
\end{array}\right]
\end{aligned}
$$

We note that $M$ is a Metzler matrix (i.e with non-negative of t-diagonal entries) in opinion of the before now recognized non-negatively of $P$. Thus an equation (3.4) is a monotone system [46]. Therefore, $R_{+}^{3}$ invariant under the flow system (3.4). This complete the proof of the theorem.

\section{Qualitative Analysis}

By substituting the values of parameters in given system of differential equations and taking rate of change with respect to time zero, we get

$$
\begin{gathered}
b N-\beta_{1} L P-\left(d_{1}+\mu\right) P+\tau Q=0, \\
\beta_{1} L P-\beta_{2} L S-\left(d_{2}+\mu\right) L=0,
\end{gathered}
$$




$$
\begin{gathered}
\beta_{2} L S-\left(\gamma+d_{3}+\mu\right) S=0, \\
\gamma S-\left(\tau+d_{4}+\mu\right) Q=0 \\
(b-\mu) N-\left(d_{1} P+d_{2} L+d_{3} S+d_{4} Q\right)=0,
\end{gathered}
$$

By solving equations. $(4.1-4.5)$, we get the disease free equilibrium point $E_{0}=(P, L, S, Q)$ i.e $E_{0}=$ $(0,0,0,0)$, which is trivial solutions. If $\beta_{1}<d_{2}+\mu$ then the disease free equilibrium point $E_{1}=\left(P_{0}, L_{0}, S_{0}, Q_{0}\right)$ i.e $E_{1}=(1,0,0,0)$

Solving the system of Equations $(4.1-4.5)$, we get

$$
E_{0}^{*}=\left(P^{*}, L^{*}, S^{*}, Q^{*}\right)
$$

where

$$
\begin{gathered}
P^{*}=\frac{\mu+d_{2}}{\beta_{1}}+\frac{\beta_{1} d_{2}\left(\mu+\gamma+d_{3}\right)-(b-\mu)\left(\mu+d_{2}\right)\left(-\beta_{2}\left(\mu+d_{1}-b d_{1}\right)+\beta_{1}\left(\mu+\gamma+d_{3}\right)\right)}{\beta_{1}\left[\beta_{1} \gamma \tau+(b-\mu)\left(-\beta_{2}\left(\mu+d_{1}+b d_{1}\right)+\beta_{1}\left(\mu+\gamma+d_{3}+d_{4}+d_{3}\left(\mu+\tau+d_{4}\right)\right)\right]\right.} \\
L^{*}=\frac{\mu+\gamma+d_{3}}{\beta_{2}} \\
S^{*}=\frac{\beta_{1} d_{2}\left(\mu+\gamma+d_{3}\right)-(b-\mu)\left(\mu+d_{2}\right)\left(-\beta_{2}\left(\mu+d_{1}-b d_{1}\right)+\beta_{1}\left(\mu+\gamma+d_{3}\right)\right)}{\beta_{2}\left[\beta_{1} \gamma \tau+(b-\mu)\left(-\beta_{2}\left(\mu+d_{1}+b d_{1}\right)+\beta_{1}\left(\mu+\gamma+d_{3}\right)\right)+\beta_{1} d_{4}+\beta_{1} d_{3}\left(\mu+\tau+d_{4}\right)\right]} \\
Q^{*}=\frac{\gamma\left[\beta_{1} d_{2}\left(\mu+\gamma+d_{3}\right)-(b-\mu)\left(\mu+d_{2}\right)\left(-\beta_{2}\left(\mu+d_{1}-b d_{1}\right)+\beta_{1}\left(\mu+\gamma+d_{3}\right)\right)\right]}{\beta_{2}\left(\mu+\tau+d_{4}\right)\left[\beta_{1} \gamma \tau+(b-\mu)\left(-\beta_{2}\left(\mu+d_{1}+b d_{1}\right)+\beta_{1}\left(\mu+\gamma+d_{3}+d_{4}+d_{3}\left(\mu+\tau+d_{4}\right)\right)\right]\right.}
\end{gathered}
$$

Theorem 3: $E_{0}$ of the given system is locally asymptotically stable if $\operatorname{Re}(\lambda)<0$.

Proof: $\lambda$ can be evaluated from the relation $|J-\lambda I|=0$. Consider the jacobian matrix again as

$$
\begin{aligned}
& J=\left[\begin{array}{cccc}
-\beta_{1} L-\left(d_{1}+\mu\right) & -\beta_{1} P & 0 & \tau \\
\beta_{1} L & \beta_{1} P-\beta_{2} S-\left(d_{2}+\mu\right) & -\beta_{2} L & 0 \\
0 & \beta_{2} S & \beta_{2} L-\left(\gamma+d_{3}+\mu\right) & 0 \\
0 & 0 & \gamma & -\tau-d_{4}-\mu
\end{array}\right] \\
& J=\left[\begin{array}{cccc}
-d_{1}-\mu & 0 & 0 & \tau \\
0 & -\left(d_{2}+\mu\right) & 0 & 0 \\
0 & 0 & -\gamma-d_{3}-\mu & 0 \\
0 & 0 & \gamma & -\tau-d_{4}-\mu
\end{array}\right]
\end{aligned}
$$


The Eigne values of the above matrix according to the equilibrium point $E_{0}=(0,0,0,0)$ are $-\left(d_{1}+\mu\right)$, $-\left(d_{2}+\mu\right),-\left(\gamma+d_{3}+\mu\right),-\left(\tau+d_{4}+\mu\right)$ with negative real parts represents that the given system is locally asymptotically stable. Also the system is stable for the point $E_{1}=(1,0,0,0)$.

Theorem 4: The The disease free equilibrium $E_{0}=(1,0,0,0,0)$ of subsystem $(2.1-2.4)$ is globally asymptotically stable (GAS) if $R_{0}<1$.

Proof:Let $P_{0}=1$ and consider the following combination of linear function and voltera type lyapunove function

$$
M_{0}=M_{0}(P, L, S, Q)=P-P_{0} \ln (P)+L+b_{1} S+b_{2} Q
$$

using the function that $P_{0}=1$, lie derivative of $M_{0}$ in the direction of vector field given by the right hand side of equation $(2.1-2.4)$ is

$$
\begin{array}{r}
\frac{d M_{0}}{d t}=\frac{d P}{d t}\left(1-\frac{1}{P}\right)+\frac{d L}{d t}+b_{1} \frac{d S}{d t}+b_{2} \frac{d Q}{d t} \\
\frac{d M_{0}}{d t}=\left(b N-\beta_{1} L P-\left(d_{1}+\mu\right) P+\tau Q\right)\left(1-\frac{1}{P}\right)+\beta_{1} L P-\beta_{2} L S-\left(d_{2}+\mu\right) L+b_{1}\left(\beta_{2} L S\right. \\
\left.-\left(\gamma+d_{3}+\mu\right)\right) S+b_{2}\left(\gamma S-\left(\tau+d_{4}+\mu\right)\right) Q \\
\frac{d M_{0}}{d t}=-\frac{b N}{P}(1+\tau Q-P)+\left(\beta_{1}+\left(b_{1}-1\right) \beta_{2} S-\left(d_{2}+\mu\right)\right) L+\left(b_{2} \gamma-b_{1}\left(\gamma+d_{3}+\mu\right)\right) S \\
+\left(\tau-b_{2}\left(\tau+d_{4}+\mu\right)\right) Q
\end{array}
$$

choose $b_{1}, b_{2}$

$$
b_{2} \gamma-b_{1}\left(\gamma+d_{3}+\mu\right)=0
$$

and

$$
\tau-b_{2}\left(\tau+d_{4}+\mu\right)=0
$$

we get

$$
\begin{gathered}
b_{2}=\frac{\tau}{\tau+d_{4}+\mu} \\
b_{1}=\frac{\tau \gamma}{\left(\tau+d_{4}+\mu\right)\left(\gamma+d_{3}+\mu\right)}
\end{gathered}
$$

with in this mind $\frac{d M_{0}}{d t}$ becomes

$$
\begin{array}{r}
\frac{d M_{0}}{d t}=-\frac{b N}{P}(1+\tau Q-P)+\left(\beta_{1}+\left(\frac{\tau \gamma}{\left(\tau+d_{4}+\mu\right)\left(\gamma+d_{3}+\mu\right)}-1\right) \beta_{2} S-\left(d_{2}+\mu\right)\right) L \\
\frac{d M_{0}}{d t}=-\frac{b N}{P}(1+\tau Q-P)+\left(\beta_{1}\left(\tau+d_{4}+\mu\right)\left(\gamma+d_{3}+\mu\right)+\left(\tau \gamma-\left(\tau+d_{4}+\mu\right)\left(\gamma+d_{3}+\mu\right)\right) \beta_{2} S\right. \\
\left.-\left(d_{2}+\mu\right)\left(\tau+d_{4}+\mu\right)\left(\gamma+d_{3}+\mu\right)\right) \leq 0
\end{array}
$$

since it is easy to see that the largest invariant subset contained in the set.

$$
\varepsilon=\left\{(P, L, S, Q) \epsilon K_{0} / \frac{d M_{0}}{d t}=0\right\}
$$




\section{Reproductive Number}

Consider the jacobian matrix as

$$
J=\left[\begin{array}{cccc}
-\beta_{1} L-\left(d_{1}+\mu\right) & -\beta_{1} P & 0 & \tau \\
\beta_{1} L & \beta_{1} P-\beta_{2} S-\left(d_{2}+\mu\right) & -\beta_{2} L & 0 \\
0 & \beta_{2} S & \beta_{2} L-\left(\gamma+d_{3}+\mu\right) & 0 \\
0 & 0 & \gamma & -\left(\tau+d_{4}+\mu\right)
\end{array}\right] .
$$

Since the Jacobian matrix is $J=F-V$ then the matrix $F$ and $V$ can be written as

$$
\begin{gathered}
F=\left[\begin{array}{cccc}
0 & -\beta_{1} & 0 & 0 \\
0 & \beta_{1} & 0 & 0 \\
0 & 0 & 0 & 0 \\
0 & 0 & 0 & 0 \\
& &
\end{array}\right], \\
V=\left[\begin{array}{cccc}
\beta_{1} L+\left(d_{1}+\mu\right) & \beta_{1} P & 0 & -\tau \\
-\beta_{1} L & -\beta_{1} P+\beta_{2} S+\left(d_{2}+\mu\right) & \beta_{2} L & 0 \\
0 & -\beta_{2} S & -\beta_{2} L+\left(\gamma+d_{3}+\mu\right) & 0 \\
0 & 0 & -\gamma & \left(\tau+d_{4}+\mu\right)
\end{array}\right] .
\end{gathered}
$$

We know that $K=F V^{-1}$ and using the relation $|K-\lambda I|=0$ for the eigen value $\lambda$, we get

$$
\lambda=\frac{\beta_{1}}{\mu+d_{2}},
$$

which shows the reproductive number $R_{0}=\frac{\beta_{1}}{\mu+d_{2}}$. By substituting the values of parameters, we get $R_{0}=$ $0.020408<1$. Since reproductive number $R_{0}<1$, so the constructed system is in disease free state.

\section{Sensitivity Analysis of $R_{0}$}

The sensitivity of $R_{0}=\frac{\beta_{1}}{\mu+d_{2}}$, to each of its parameters is

$$
\begin{gathered}
\frac{\partial R_{0}}{\partial \beta_{1}}=\frac{1}{\mu+d_{2}} \geq 0 \\
\frac{\partial R_{0}}{\partial \mu}=-\frac{\beta_{1}}{\left(\mu+d_{2}\right)^{2}} \leq 0 \\
\frac{\partial R_{0}}{\partial d_{2}}=-\frac{\beta_{1}}{\left(\mu+d_{2}\right)^{2}} \leq 0
\end{gathered}
$$


It can be seen that $R_{0}$ is most sensitive to change in parameter, here, $R_{0}$ is increasing with $\beta_{1}$ and decreasing with $\mu, d_{2}$. In other words it found that the sensitivity analysis shows that prevention is better than to control the disease.

TABLE 1. Values of physical parameters used in smoking model

\begin{tabular}{|c|c|c|c|}
\hline Parameter & Value & Parameter & Value \\
\hline$n_{1}$ & 40 & $n_{2}$ & 40 \\
\hline$n_{3}$ & 60 & $n_{4}$ & 80 \\
\hline$n_{5}$ & 200 & $d_{1}$ & 0.33 \\
\hline$d_{2}$ & 0.44 & $d_{3}$ & 0.55 \\
\hline$d_{4}$ & 0.66 & $\beta_{1}$ & 0.001 \\
\hline$\beta_{2}$ & 0.001 & $\mu$ & 0.05 \\
\hline$b$ & 0.1 & $\gamma$ & 0.99 \\
\hline$\tau$ & 0.2 & & \\
\hline
\end{tabular}

\section{Nonstandard finite difference (NSFD) SCheme}

A nonstandard finite difference (NSFD) scheme for the system $(2.1-2.5)$ is presented in this section [29]. In recent years, nonstandard finite difference (NSFD) scheme for discrete models have been constructed or tested for a wide range of nonlinear systems of differential equations [30-32]. The positivity of the state variables involved in the system is satisfy by proposed method. This property has key role when we solve mathematical models arising in biology because these state variables represent sub-populations which never take negative values. The discretized form of the the system $(2.1-2.5)$ by using NSFD scheme which based on the generalized first order forward method is written as

$$
\begin{gathered}
\frac{P^{k+1}-P^{k}}{\phi}=b N^{k}-\beta_{1} L^{k} P^{k+1}-\left(d_{1}+\mu\right) P^{k+1}+\tau Q^{k} \\
P^{k+1}+\beta_{1} L^{k} P^{k+1} \phi+\phi\left(d_{1}+\mu\right) P^{k+1}=P^{k}+b \phi N^{k}+\tau \phi Q^{k} \\
P^{k+1}=\frac{P^{k}+b \phi N^{k}+\tau \phi Q^{k}}{1+\beta_{1} L^{k} \phi+\phi\left(d_{1}+\mu\right)} \\
\frac{L^{k+1}-L^{k}}{\phi}=\beta_{1} L^{k} P^{k+1}-\beta_{2} L^{k+1} S^{k}-\left(d_{2}+\mu\right) L^{k+1} \\
L^{k+1}+\phi \beta_{2} L^{k+1} S^{k}+\phi\left(d_{2}+\mu\right) L^{k+1}=L^{k}+\beta_{1} L^{k} P^{k+1} \\
L^{k+1}=\frac{L^{k}+\beta_{1} L^{k} P^{k+1}}{1+\phi \beta_{2} S^{k}+\phi\left(d_{2}+\mu\right)}
\end{gathered}
$$




$$
\begin{gathered}
\frac{S^{k+1}-S^{k}}{\phi}=\beta_{2} L^{k+1} S^{k}-S^{k+1}\left(\gamma+d_{3}+\mu\right) \\
S^{k+1}=\beta_{2} L^{k+1} S^{k} \phi-\phi S^{k+1}\left(\gamma+d_{3}+\mu\right) \\
S^{k+1}\left(1+\phi\left(\gamma+d_{3}+\mu\right)\right)=\beta_{2} L^{k+1} S^{k} \phi+S^{k} \\
S^{k+1}=\frac{\beta_{2} L^{k+1} S^{k} \phi+S^{k}}{1+\phi\left(\gamma+d_{3}+\mu\right)} \\
Q^{k+1}-Q^{k}=\phi \gamma S^{k+1}-\phi\left(\tau+d_{4}+\mu\right) Q^{k+1} \\
Q^{k+1}\left(1+\phi\left(\tau+d_{4}+\mu\right)\right)=\phi \gamma S^{k+1}+Q^{k} \\
Q^{k+1}=\frac{\phi \gamma S^{k+1}+Q^{k}}{1+\phi\left(\tau+d_{4}+\mu\right)}
\end{gathered}
$$

which is the purposed NSFD scheme for the given model, where

$$
\phi=\phi(h)=\frac{1-e^{-\left(d_{3}+\mu+\gamma\right) h}}{d_{3}+\mu+\gamma}
$$

The discrete method given in $(22,25,29,32)$ is indeed an NSFD scheme because it is constructed according to Mickens rules [32] formalized as follows in [33]. Rule 1. The standard denominator $h=\Delta t$ of the discrete derivatives is replaced by the complex denominator function in Equation (33) which satisfies the asymptotic relation

$$
\phi(h)=h+O\left(h^{2}\right)
$$

Note that the denominator function $\phi$ is expected to better capture the dynamics of the continuous model through the presence of the underlying parameters $d_{3}, \mu, \gamma$. In fact, exact schemes for a wide range of dynamical systems involve such complex denominator functions [34,35]. Rule 2. Nonlinear terms in the right-hand side of Equation $(2.1-2.5)$ are approximated in a non-local way. For instance, we have $L_{t_{k}} P_{t_{k}} \simeq$ $L_{k} P_{k+1}$ instead of $L_{t_{k}} S_{t_{k}} \simeq L_{k} P_{k}$

\section{Analysis of the SCheme}

Theorem 5: The NSFD scheme $(22,25,29,32)$ is a dynamical system on the biological feasible domain $\mathcal{K}$ of the continuous model $(2.1-2.5)$.

Proof: First, we prove the positivity of the scheme $(22,25,29,32)$. It is easy to show that the NSFD scheme $(22,25,29,32)$ takes the explicit form

$$
P^{k+1}=\frac{P^{k}+b \phi N^{k}+\tau \phi Q^{k}}{1+\beta_{1} L^{k} \phi+\phi\left(d_{1}+\mu\right)}
$$




$$
\begin{gathered}
L^{k+1}=\frac{L^{k}\left(1+\beta_{1} L^{k} \phi+\phi\left(d_{1}+\mu\right)\right)+\left(\beta_{1} L^{k}\right)\left(P^{k}+b \phi N^{k}+\tau \phi Q^{k}\right)}{\left(1+\phi \beta_{2} S^{k}+\phi\left(d_{2}+\mu\right)\right)\left(1+\beta_{1} L^{k} \phi+\phi\left(d_{1}+\mu\right)\right)} \\
S^{k+1}=\frac{\beta_{2} S^{k} \phi A+S^{k} B}{\left(1+\phi\left(\gamma+d_{3}+\mu\right)\right) B} \\
Q^{k+1}=\frac{\phi \gamma\left(\beta_{2} S^{k} \phi A+S^{k} B\right)+Q^{k}\left(1+\phi\left(\gamma+d_{3}+\mu\right)\right) B}{\left(1+\phi\left(\tau+d_{4}+\mu\right)\right)\left(1+\phi\left(\gamma+d_{3}+\mu\right)\right) B}
\end{gathered}
$$

where

$$
A=L^{k}\left(1+\beta_{1} L^{k} \phi+\phi\left(d_{1}+\mu\right)\right)+\left(\beta_{1} L^{k}\right)\left(P^{k}+b \phi N^{k}+\tau \phi Q^{k}\right), B=\left(1+\phi \beta_{2} S^{k}+\phi\left(d_{2}+\mu\right)\right)\left(1+\beta_{1} L^{k} \phi+\phi\left(d_{1}+\mu\right)\right)
$$

Thus $P^{k+1} \geq 0, L^{k+1} \geq 0, S^{k+1} \geq 0, Q^{k+1} \geq 0$ whenever the discrete variables are non-negative at the previous iteration. It remains to prove the positive invariance of $\mathcal{K}$. Adding the $(22,25)$ and $(29)$ we have

$$
\begin{gathered}
{\left[1+\phi\left(d_{1}+\mu\right)\right] H^{k+1}=\phi b N+H^{k}-\left[1+\left(d_{2}+\mu\right) \phi\right] L^{k+1}-\left[1+\phi\left(\gamma+d_{3}+\mu\right)\right] S^{k+1} \leq \phi b N+H^{k}} \\
{\left[1+\phi\left(\mu+d_{1}\right)\right] H^{k+1} \leq \phi b N+H^{k}} \\
\Rightarrow H^{k+1} \leq \frac{b N}{d_{1}+\mu}
\end{gathered}
$$

whenever

$$
H^{k} \leq \frac{b N}{\mu+d_{1}}
$$

The priori bonds for $Q^{k+1}$ and $N^{k+1}$ follow the radially from the fact that $L^{k+1}$ and $S^{k+1}$ are less then or equal to $H^{k+1}$. This complete the proof.

\section{Numerical Simulations}

The mathematical analysis of smoking epidemic model with non-linear incidence has been presented. Firstly, we investigate the basic reproduction number $R_{0}$ for the system $(2.1-2.5)$ which completely characterized the stability of the disease free and endemic equilibrium. We observed that, if $R_{0}<1$, the disease free state at $E_{0}$ and $E_{1}$ is locally stable. To observe the effects of the parameters using in this dynamics smoking model $(2.1-2.5)$, conclude several numerical simulations varying the value of parameters. These simulations reveals that a change in time and step size $h$ affects the dynamics of the epidemic as shown in Figures 1 and Figures 2. By applying the Mickens approach, we use $\phi=\phi(h)$ instead of step size $h$ in figure 2 and figure 4 . comparison is made by highlighting the point in each graph which shows that the Smokers reduces within 20 weeks when we used $\phi$. Its interpretation for a longer period reduces the infected individuals in the health system. When initial condition changes to $P(0)=40, L(0)=80, S(0)=120, Q(0)=160, N(0)=400$, the convergence to disease free equilibrium point remain consistent as shown in figure 3 and Figures 4 . 


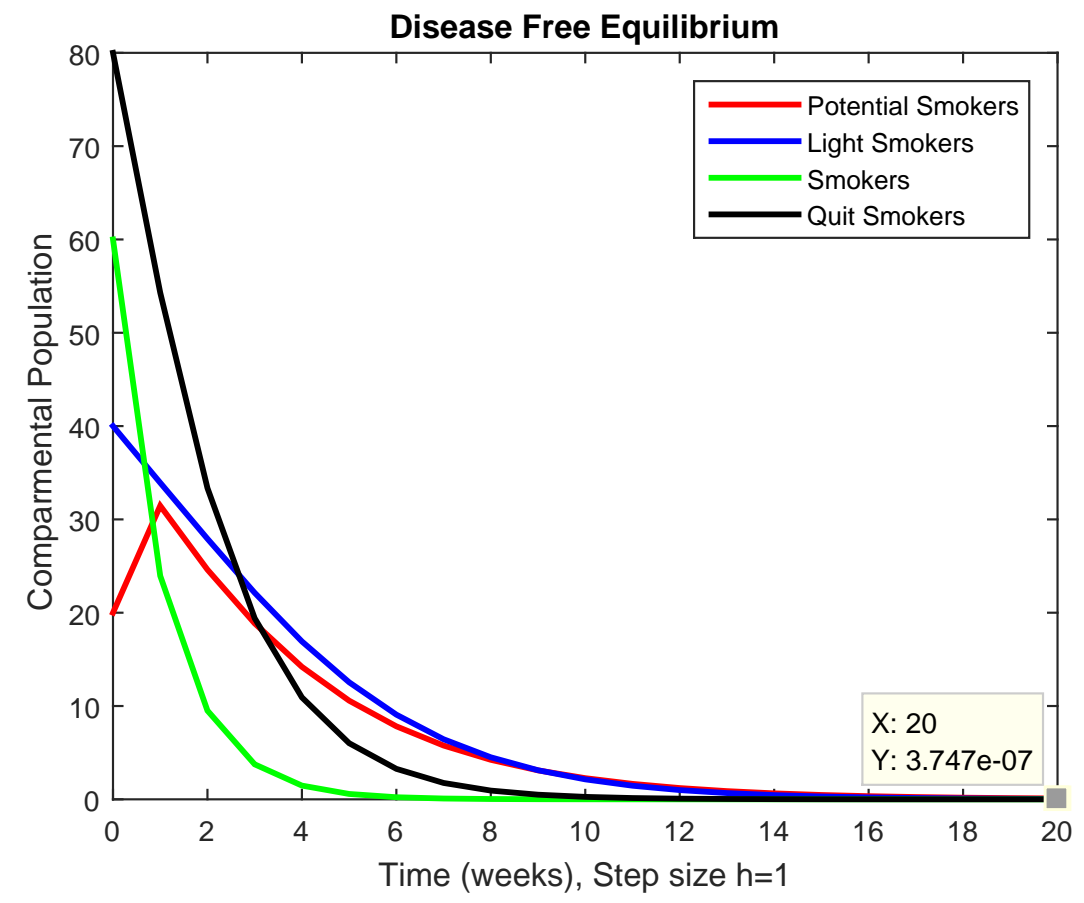

Figure 1. Numerical solutions for potential smokers, Light Smokers, Smokers and Quit Smokers in a time $t$ (weeks) with step size $h=1$

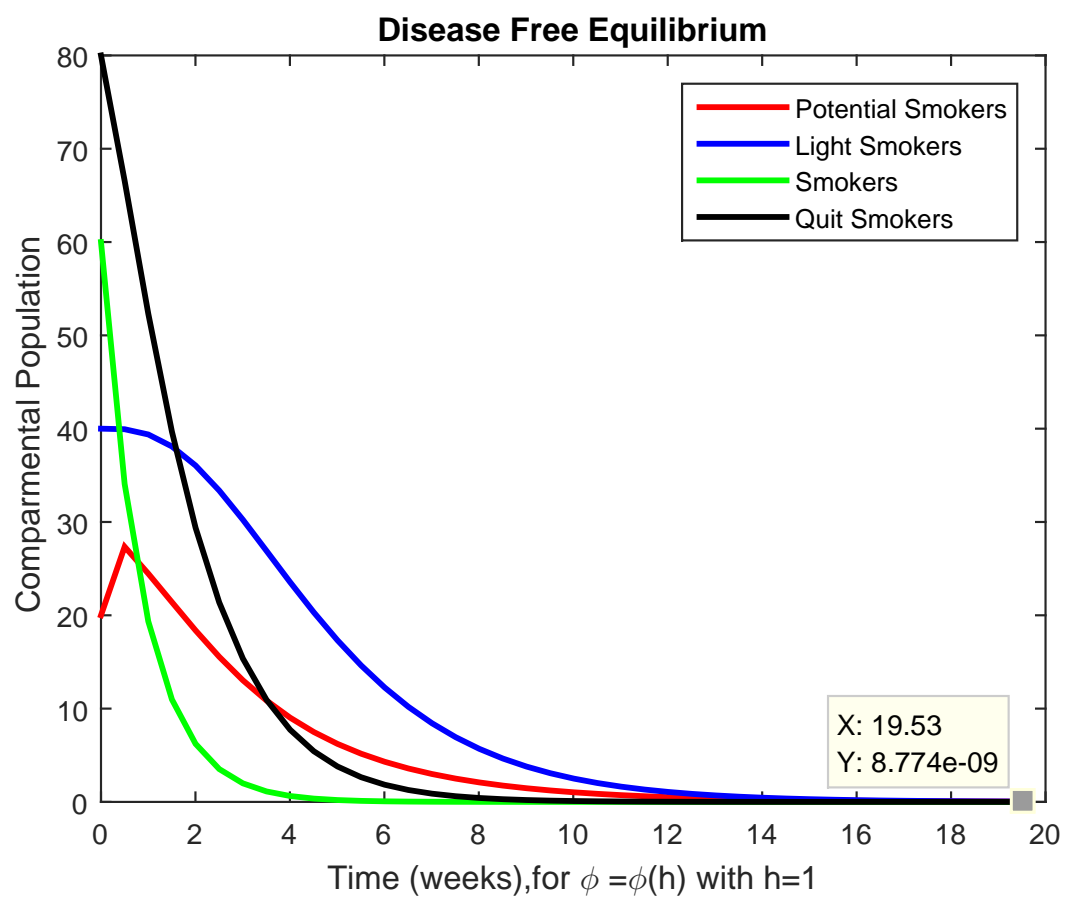

Figure 2. Numerical solutions for potential smokers, Light Smokers, Smokers and Quit Smokers in a time $t$ (weeks)for $\phi=\phi(h)$ with $h=1$ 


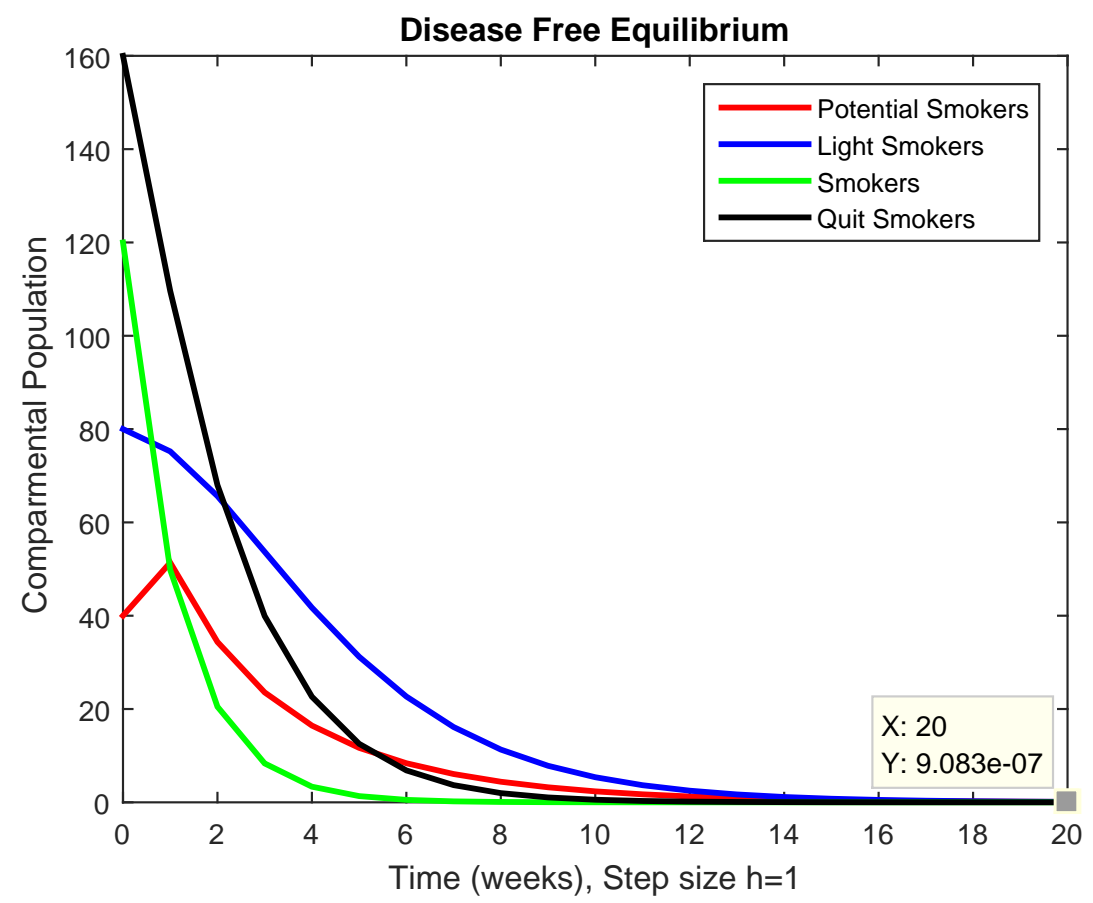

Figure 3. Numerical solutions for potential smokers, Light Smokers, Smokers and Quit Smokers in a time $t$ (weeks) with step size $h=1$ with different initial conditions

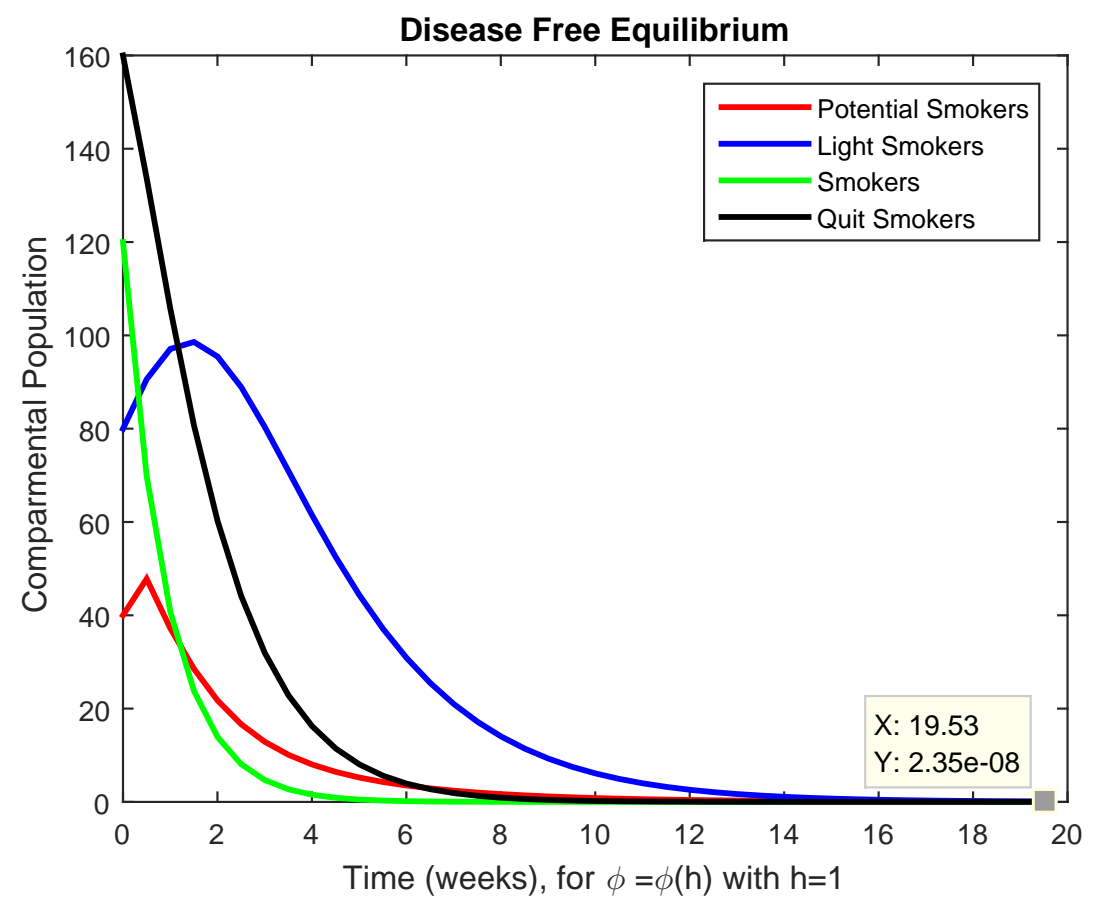

Figure 4. Numerical solutions for potential smokers, Light Smokers, Smokers and Quit Smokers in a time $t$ (weeks) for $\phi=\phi(h)$ with $h=1$ with different initial conditions 


\section{Conclusion}

It is an important to note that nonstandard finite difference scheme for mathematical models based on system of differential equations is more powerful approach to compute the convergent solution. The constructed unconditionally convergent nonstandard finite difference (NSFD) scheme for smoking model preserve the positivity of all values of $h$ (step size) which shows that the developed Scheme is stable. The nonstandard finite difference scheme is dynamically consistent, easy to implement and shows a good agreement to analyze the bad impact of smoking for long period of time and represents their dynamical behavior graphically. Threshold condition shows most sensitive effect regarding their parameters. We prove the essential properties, bounded, positivity and well-posed, also local and global stability analysis has been made to analyze the smoking effects in the community. Numerical simulations are carried out to check the actual behavior of the model.

\section{References}

[1] J. Biazar, Solution of the epidemic model by Adomian decomposition method, Appl. Math. Comput. 173 (2006), $1101-1106$.

[2] S. Busenberg and P. Driessche, Analysis of a disease transmission model in a population with varying size, J. Math. Biol. 28 (1990), 65-82.

[3] A.M.A. El-Sayed, S.Z. Rida and A.A.M. Arafa, On the solutions of time-fractional bacterial chemotaxis in a diffusion gradient chamber, Int. J. Nonlinear Sci. 7 (2009), 485-495.

[4] A.A.M. Arafa, S.Z. Rida and M. Khalil, Fractional modeling dynamics of HIV and 4 T-cells during primary infection, Nonlinear Biomed. Phys. 6 (2012), 1-7.

[5] C.M. Kribs-Zaleta, Structured models for heterosexual disease transmission, Math. Biosci. 160 (1999), 83-108.

[6] B. Buonomo and D. Lacitignola, On the dynamics of an SEIR epidemic model with a convex incidence rate, Ricerche Mat. 57 (2008), 261-281.

[7] X. Liu and C. Wang, Bifurcation of a predator-prey model with disease in the prey, Nonlinear Dyn. 62 (2010), 841-850.

[8] F. Haq, K. Shah, G.U Rahman and M. Shahzad. Numerical solution of fractional order smoking model via laplace Adomian decomposition method, Alex. Eng. J. 57 (2018), 1061-1069.

[9] C. Chavez and B. Song; Dynamical models of tuberculosis and their applications; Math. Biosci. Eng. 1 (2004), 361-404.

[10] A. McNeill, M. Raw, J. Whybrow and P. Bailey; National strategy for smoking cessation treatment in England; Addiction 100 (S.2) (2005), 1-11.

[11] R.P. Sargent, R.M. Shepard and S.A. Glantz; Admission for myocardial infarction associated with public smoking bun; Br.Med. J. 1 (2004), 328-977.

[12] Y.M. Terry-McElrath, M.A. Wakefield, S. Emery, H. Saffer, G.M. Szczypka and P. O. Malley P; State antitobacco advertising and smoking outcomes by gender and race/ethnicity; Ethnicity and Health 12 (2007), 339-362.

[13] R. Ullah, M. Khan, G. Zaman, S. Islam, M.A. Khan, S. Jan and T. Gul, Dynamical Featurers of mathemtical model on smoking, J. Appl. Environ. Biol. Sci., 6 (2016), 92-96.

[14] http://en.wikipedia.org/wiki/Smoking (3th October, 2016).

[15] https://en.wikipedia.org/wiki/Epidemiology (17th November, 2016). 
[16] C. Castillo-Garsow, G. Jordan-Salivia, and A. Rodriguez Herrera, Mathematical models for the dynamics of tobacco use, recovery, and relapse, Technical Report Series BU-1505- M, Cornell University, Ithaca, NY, USA, (1997).

[17] O. Sharomi and A. B. Gumel, Curtailing smoking dynamics: A mathematical modeling approach, Appl. Math. Comput. 195 (2008), 475-499.

[18] G. Zaman, Qualitative behavior of giving up smoking model; Bull. Malaysian Math. Sci. Soc. 2 (2011), $403-415$.

[19] S.A. Matintu, Smoking as Epedemic: Modeling and Simulation study, American J. Appl. Math. 5 (2017), 31-38.

[20] A. Ahmad, M. Farman, F. Yasin and M. O. Ahmad, Dynamical transmission and effect of smoking in society, Int. J. Adv. Appl. Sci. 5(2) (2018), 71-75

[21] F. Ashraf, A. Ahmad, M. U. Saleem, M. Farman and M.O. Ahmad, Dynamical behavior of HIV immunology model with non-integer time fractional derivatives, Int. J. Adv. Appl. Sci. 5(3) (2018), 39-45, .

[22] A. Ahmad, M. Farman, M. O Ahmad, N. Raza and Abdullah, Dynamical behavior of SIR epidemic model with non-integer time fractional derivatives: A mathematical analysis, Int. J. Adv. Appl. Sci. 5(1) (2018), 123-129.

[23] J.B. Swartz, Use of a multistage model to predict time trends in smoking induced lung cancer, J. Epidemiol. Commun. Healt. 46 (1992), 11-31.

[24] F. Brauer and C. Castillo-Cha vez, Mathematical Models in Population Biology and Epidemiology, Springer, (2001).

[25] A. Zeb, G. Zaman, V.S. Erturk, B. Alzalg, F. Yousafzai and M. Khan, Approximating a giving up smoking dynamic on adolescent nicotine dependence in fractional order, PLoS ONE, 11 (2016), 10-15.

[26] G. Zaman, Optimal campaign in the smoking dynamics, Comput. Math. Method. Med. 2011 (2011), Article ID 163834.

[27] G. Zaman, Qualitative behavior of giving up smoking models, Bull. Malay. Math. Sci. Soc. 34 (2011), 403-415.

[28] V. Suat Erturk, G. Zamanb and S. Momanic, A numeric analytic method for approximating a giving up smoking model containing fractional derivatives, Comput. Math. Appl. 64 (2012), 3065-3074.

[29] R. E. Mickens, Exact solutions to a finite difference model of a nonlinear reactions advection equation: Implications for numerical analysis, Numer. Methods Partial Differ. Equations, 5 (1989), 313-325.

[30] R. E.Mickens, Applications of Nonstandard finite difference Schemes, World Scientific, Singaporen (2000).

[31] R. Anguelov and J. M.-S. Lubuma, Nonstandard finite difference method by nonlocal approximations, Math. Comput. Simul. 61 (2003), 465-475.

[32] R. E. Mickens, Nonstandard finite difference Models of differential equations, World Scientific, Singapore (1994).

[33] R. Anguelov and J.M.-S. Lubuma, Contributions to the mathematics of the nonstandard nite dierencemethodandapplications, Numer. Methods Partial Differ. Equations, 17 (2001), 518-543.

[34] J.M.-S. Lubuma and K.C. Patidar, Non-standard methods for singularly perturbed problems possessing oscillatory/layer solutions, Appl. Math. Comput. 187(2) (2007), 1147-1160.

[35] L.W. Roeger, Exact difference schemes, in A. B. Gumel Mathematics of Continuous and Discrete Dynamical Systems, Contemp. Math. 618 (2014), 147-161. 